\title{
Randomized trials in robotic surgery: a practical impossibility?
}

\author{
Sarah Collins $\cdot$ Paul Tulikangas
}

Received: 11 May 2010 / Accepted: 28 June 2010 / Published online: 14 July 2010

(C) The International Urogynecological Association 2010

An eager new fellow in female pelvic medicine and reconstructive surgery, I began training at Hartford Hospital in July of 2008. Arriving at an institution with four Da Vinci robots, I quickly became aware of the controversy surrounding the relatively recent emergence of this technology. I heard the complaints of the disgruntled general surgeons, gynecologists, and urologists forced to question their tried and true techniques in the face of growing pressures driven by industry, institution, and even by patients. In contrast, I appreciated the excitement of surgical subspecialists confident that this technology would revolutionize their methods. Skeptics cited the astronomic costs, lack of haptic feedback, and risks associated with prolonged steep Trendelenburg position as potential pitfalls of the Da Vinci system. Champions of the robot touted its improved ergonomics, three-dimensional visual properties, shorter hospitalization, and decreased analgesic requirements as primary advantages. A common underlying theme to all of the debate was a fundamental lack of answers: there is scant quality evidence to guide judgment for or against the robot.

Armed with energy, interest, and just enough naivety, I designed a study that would provide much-needed answers. I would enroll urogynecology patients undergoing sacral colpopexy and randomize them to the abdominal or robotic approach. Using accelerometers, tiny devices that measure their wearers' movement in all three dimensions and record how vigorous that movement is, I would compare how quickly patients return to their baseline physical activity

S. Collins $(\bowtie) \cdot$ P. Tulikangas

Female Pelvic Medicine and Reconstructive Surgery,

Department of Obstetrics and Gynecology, Hartford Hospital,

85 Seymour St., Ste. 525,

Hartford, CT 06106, USA

e-mail: scollins@harthosp.org after surgery by these two approaches. I wrote my protocol, and the IRB approved it. I applied for funding and eventually got some. I excitedly made my sealed, numbered, opaque envelopes. My attendings voiced their enthusiasm and support, and I began to recruit patients.

Enter reality. Most patients politely declined information about my study and asserted their preference for the robotic approach. Some were intrigued enough to accept written information about the project and agreed to meet with me. These women listened to my description of the study, held my precious accelerometers in their hands, and asked whether they could decline participation in the study if they were randomized to laparotomy. Although my frustration grew, I remained hopeful that things would turn around...until one fateful day, when I discovered that they wouldn't. In clinic with one of my attendings, I saw a healthy, sexually active patient with vault prolapse who wanted definitive surgery, a perfect candidate for my trial. She voiced concerns about getting back to work quickly. When my attending told her she should have robotic surgery to get back on her feet faster, I was forced to accept that the problem of bias extends to physicians as well as patients.

Our conservative power analysis projected an enrollment goal of 50 subjects. Over 6 months, I approached 100 subjects and enrolled and randomized three. Ironically, each of these women was randomized to laparotomy. After changing the protocol to a prospective cohort study design, I called each of my three randomized subjects and explained that the study they had entered had changed. They were released from their obligations to undergo laparotomy. All three promptly rescheduled their surgeries to be done robotically.

Still enthusiastic about my work and the results I hope it yields, I also feel mildly defeated. I won't be increasing the 
volume of level I evidence to inform the use of robotic surgery in urogynecology, though I had hoped to. Reflecting on the forces that have stood in my way, I have identified three primary sources of resistance to my study and others like it.

Patients may be the strongest force against evidence when it comes to robotic surgery. Products and procedures which are "new" carry an allure for patients regardless of what else they may have to offer. A recent study demonstrated this phenomenon in women who were offered "new" vs. "old" procedures for stress urinary incontinence; when the rectus fascia sling was touted as the "new option," it was chosen by 22 of 24 subjects over the "old" synthetic mesh sling. When the rectus fascia sling was called "old" and the mesh sling "new," 13 of 23 subjects selected it, a significant difference. Overall, almost $80 \%$ of these women stated that they considered newer surgical procedures to be better than older ones [1]. This bias toward the new may compound the intrigue of the robot, with which many of our patients may already be familiar thanks to frequently aired commercials for hospitals and popular medical TV dramas like "Gray's Anatomy." The effect of the robot's fame has been felt by several of our urology colleagues, as documented in a recent New York Times article [2]. In it, a 42-year-old laparoscopically trained urologist describes feeling forced to offer robotic prostatectomy. "Patients interview you. They say, 'Do you use the robot? OK, well, thank you.' And then they leave." He added, "The battle is lost. Marketing is driving the case here."

Whatever is actually driving patients to choose robotic surgery, it is not scientific evidence. A review of the Cochrane Central Registry of Controlled Trials reveals disappointingly few randomized trials on robotic surgery in gynecology, general surgery, or urology. The largest three of five published studies randomized 50 subjects. All studies showed no obvious difference between various short-term outcome measures in robotic versus traditional laparoscopy, but none are sufficiently powered to show equivalence or even non-inferiority of the robotic approach. There is one ongoing study of robotic or laparoscopic vs. abdominal radical hysterectomy which will be powered for equivalence in disease-free survival [3], but the grouping together of robotic and laparoscopic procedures will make it difficult to assess whether the robotic approach offers any particular advantage. A search of clinicaltrials.gov reveals six randomized trials of robotics in urology, gynecology, and general surgery. Two of these (NCT00551993, NCT 00895284) are specific to gynecology and, when published, may stand alone as true sources of level I evidence in gynecologic surgery using the Da Vinci robot.

Physician bias also poses a barrier to quality evidence in robotic surgery. Just as my attending did, many of us assume that the same advantages seen with laparoscopic surgery when compared to abdominal surgery will be seen in robotic surgery. These assumptions may be valid - the incisions are similar, and so may be the blood loss and operative times. We may discharge robotic surgery patients earlier than those who undergo the same procedures through laparotomies and feel that this is a tangible advantage of the robot. It is unclear, however, whether earlier discharge is prompted by objective clinical measures of perioperative recovery or by the surgeon's or patient's expectation that recovery after robotic surgery is enhanced. We can make few assumptions, however, about the advantages of robotic over laparoscopic surgery, especially when the higher costs of the Da Vinci procedures are considered [4].

Lastly, there are some institutional pressures that many surgeons face to perform robotic procedures. Hospitals that spend close to $\$ 2$ million to buy a Da Vinci robotic system and then commit to almost $\$ 150,000$ in annual maintenance fees are motivated to maximally utilize these devices. Surgeons credentialed to use the robot may therefore be urged to use it whenever possible. This pressure is then reinforced by minimum numbers of procedures required to maintain robotic privileges. Requiring surgeons to perform a minimum number of procedures might improve efficiency and safety, but it also may influence surgeons to select the robotic approach for reasons other than empirical evidence that the robot provides betterquality care.

We practice in an era when technology outruns the pace of trials; some surgical novelties fall out of favor before the results of such trials are ever published. The recent urogynecology literature provides such an example in the SURx ${ }^{\circledR}$ system, a radiofrequency device used for stress urinary incontinence. After promising clinical trial results, this system became available in 2002 and was used enthusiastically for several years. Short-term data from prospective, nonrandomized trials suggested significant improvement in stress urinary incontinence symptoms [5, 6]. Longer-term data, however, were less favorable [7]. At the annual meeting of the American Urogynecologic Society in 2005, additional unfavorable data was presented, and prominent urogynecologic surgeons who once promoted the device stopped using it as a result. CooperSurgical, Inc. then stopped marketing the device in 2006 [8], though a faithful few practitioners have continued to use it. It was not until May 2006, when the trend had all but faded from clinical use, that the first data from a randomized controlled trial were published. They showed no improvement in quality of life compared to a sham procedure [9].

It is easy to be pessimistic about the ability to perform quality research when the researchers (surgeons), the subjects (patients), and the settings (hospitals) are already significantly biased. As a group, however, physicians are 
still scientists. We know the difference between evidence and anecdote, and we know why data from RCTs are so important in guiding practice. The risk of going forward without this data is that we may be wasting valuable resources without providing an advantage for our patients. This risk must be balanced with the desire of the medical community to provide what they believe, based on limited evidence, to be the best care currently available to their patients.

\section{References}

1. FitzGerald MP, Elliott C, Brubaker L (2008) New vs. old: descriptors can effect patients' surgical preferences. Am J Obstet Gynecol 199(5):476e.1-476e.3

2. Kolata G "Results Unproven, Robotic Surgery Wins Converts." The New York Times. February 14, 2010, pA1. Accessed at http:// www.nytimes.com/2010/02/14/health/14robot.html
3. Obermair A, Gebski V, Fumovitz M, Soliman PT, Schmeler KM, Levenback C, Ramirez PT (2008) A Phase III randomized clinical trial comparing laparoscopic or robotic radical hysterectomy with abdominal radical hysterectomy in patients with early stage cervical cancer. J Minim Invasive Gynecol 15(5):584-588

4. Patel M, O'Sullivan D, Tulikangas PK (2009) A comparison of costs for abdominal, laparoscopic, and robot-assisted sacral colpopexy. Int Urogynecol J 20:223-228

5. Fulmer BR, Kyoko S, Turk TMT, Galen D, Presthus JB, Abbott K, Ross J, Chau-su-ou, Albala DM (2002) J Urol 167(1):141-145

6. Dmochowski RR, Avon M, Ross J, Cooper JM, Kaplan R, Love B, Kohli N, Albala D, Shingleton B (2003) Transvaginal radio frequency treatment of the endopelvic fascia: a prospective evaluation for the treatment of genuine stress urinary incontinence. J Urol 169(3):1028-1032

7. Buchsbaum GM, McConville J, Korni R, Duecy EE (2007) Int Urogyencol J 18:263-265

8. Bates B Brakes put on SURx system for stress incontinence. ObGyn News. August 1, 2006, p18

9. Appell RA, Juma S, Wells WG, Lenihan JP, Klimberg IW, Kanellos A, Reilley SF (2006) Transurethral radiofrequency energy collagen micro-remodeling for the treatment of female stress urinary incontinence. Neurourol Urodyn 25:331-336 